\title{
Nonlinear tourist flows in Barcelona
}

\author{
Enric Trullols, Immaculada Massana, Joana d'Arc Prat, Josefina Antonijuan, \\ Gerard Olivar
}

\begin{abstract}
Tourism is not only a source of wealth, but also a positive way of knowing and mixing local and foreign cultures. However, overexploitation of natural resources and inadequate behaviours (among other factors) can lead to a conflict between tourists and locals that makes the tourist economy not sustainable. The modelling of tourist flows is a good tool to face and overcome this problem, looking for the balance between natural and socioeconomic resources, the interests and rights of tourists and locals. This paper proposes a mathematical model of nonlinear differential equations, which allows to study the dynamic interaction between the main factors that affect the tourist flows of Barcelona. We have used non-linear Ordinary Differential Equations to represent the interactions between residents, tourists and investors. Political/economic decisions and geopolitical factors have been added as external forces.
\end{abstract}

Specific aspects of Barcelona have been taken into account, such as the impossibility of extending the city, the lack of regulation (and excessive regulation), the affluence of investors and the increase in prices (which pressures residents outside from the city)

Our results show that sustainability, that is, the positive values of the population of tourists, locals and investors, in the long run is possible under the appropriate economic and social decisions.

\section{Introduction}

Barcelona, the capital of Catalonia, is one of the most popular European destinations for international tourism. The number of overnight stays in Barcelona reached 9.5 million in 2017. The international expense of visitors to Barcelona has increased progressively in recent years, in line with the growing volume of tourists arriving in the city. Passenger traffic to Barcelona airport has increased more than doubled since 2000 to reach 47.3 million by 2017 (50 million by 2018). The city is also affected by cruise tourism, with more than 700 cruises arriving at the Port of Barcelona every year. Approximately 2.7 million cruise passengers embarked, landed or moved to the port in 2017 [1, 2, 3].

Some residents are not satisfied with the strong increase in tourism and describe Barcelona as a saturated city where prices have skyrocketed and locals have trouble finding a flat at 
an affordable price. The first confrontations between tourists and locals have arrived, and the city council takes drastic measures such as the freezing of tourist licenses [4].

Dynamic interactions between populations and natural resources has been studied by several authors, starting with the predator-prey seminal work of Lotka and Volterra $[5,6]$. Brander and Taylor [7] presented a simple predatorprey model of renewable resource use which simulates the history of the Easter Island Civilisation, describing the presence of feastfamine cycles. They showed that the overexploitation of natural resources caused a sharp reduction in the human population. Several other authors have developed this model taking into account additional aspects such as institutions, property rights and technical progress [8].

\section{The model}

Regarding tourist flows and the impact on the environment, Rinaldi and Casagrandi [9] proposed a model referring to a non-specific site that includes tourism ( $\mathrm{T}$ ), environment $(\mathrm{E})$ and capital (C). Tourists (T) and capital (C) impact negatively on environmental quality (E), while environmental quality and infrastructure are attractive for tourists. The positive flow of $\mathrm{T}$ to $\mathrm{C}$ represents the investment of part of the benefits associated with tourism in new facilities for visitors.

Our work presents a model adapted to Barcelona, which includes only two actors, tourists $(\mathrm{T})$ and residents (R). In some way the model can be seen as a simplification of the model of Rinaldi and Casagrandi [9] in which the residents (R) play the role of environment (E). We have assumed that the capital (C) is proportional to the tourist ( $\mathrm{T}$ ) as follows $C=a T-b T$ where $a$ is the average spend per tourist and $b$ is related to taxes and local investments. As capital (C) is proportional to tourists $(\mathrm{T})$, it seems appropriate to proceed only with two variables ( $\mathrm{T}$ and $\mathrm{R}$ ).

Let's consider the number of tourists $\mathrm{T}(\mathrm{t})$ and residents $\mathrm{R}(\mathrm{t})$. Both are functions of time. More specifically, $\mathrm{T}$ and $\mathrm{R}$ are the number of daily overnight stays.

According to the literature [7] we have assumed that natural growth can be characterized by simple logistic dynamics. The associated equation modelizes a first step of rapid growth proportional to the population $(\mathrm{P})$ and a second step of deceleration proportional to the square of the population in which the population tends to stabilize reaching its maximum capacity.

$$
\dot{P}=\alpha P\left(1-\frac{P}{k}\right)
$$

Barcelona is one of the most densely populated cities in Europe, limited by the moun- 
tains and the sea and has no possibility to expand. Even more, Barcelona has achieved its maximum capacity (around 2 million beds including tourists and locals). This is a notorious difference with respect to other cities and this fact must be taken into account in our model.

With regard to the tourist equation (2), the first term is associated with the novelty and the attraction of the city by itself and driven by the shared positive experiences of tourists. The second term, of slowdown, is related to negative experiences of the tourists associated with the massification, price increase, loss of originality and crimes among others [10]. A new term has been added to the logistics equation to model the direct action of the residents against the tourists (or in favor).

$$
\dot{T}=\alpha_{1} T\left(1-\frac{T}{k_{1}}\right)-\alpha_{2} R
$$

where,

- $\alpha_{1}$ is the natural growth factor (the area become known by word-a-mouth recommendations of the tourists).

- $\alpha_{2}$ is a factor related to the actions and policies in favour or against the tourist.

- $k_{1}$ is the asymptotic maximum (carrying capacity).

In the absence of tourists, the population of residents $\mathrm{R}$ can also be modeled with a logistics equation. The model (3) assumes an initial growth of the population associated with new opportunities and free spaces and a process of slowdown until it reaches its maximum capacity. In presence of a parallel population of tourists, who share the same spaces in the city, the logistic equation must be modified. The first modification deals with the maximum capacity of the city, as explained above, Barcelona has no possibility to increase its capacity and the increase of tourism population implies a decrease of the residents population. A second modification deals with the fact that a small population of residents does not support the tourist pressure and tends to disappear (Alee effect). Investors mobbing against locals could be included in these Allee effect.

$$
\dot{R}=\alpha_{3} R\left(1-\frac{R}{\left(k-k_{1}\right)}\right)\left(\frac{R}{k 2}-1\right)-\alpha_{4} R T
$$

where,

- $\alpha_{3}$ is the natural growth factor (intrinsic regeneration rate). 
- $\alpha_{4}$ is a factor related to the actions and policies in favour or against the tourist.

- $k_{1}$ is the asymptotic maximum (carrying capacity).

- $k-k_{1}$ is the asymptotic maximum, being $k$ the capacity of the city.

- $k_{2}$ is the survival number (values under this tends to extinguish the population).

Consequently, the system that relates tourists to residents, is given by,

$$
\left\{\begin{aligned}
\dot{T} & =\alpha_{1} T\left(1-\frac{T}{k_{1}}\right)-\alpha_{2} R \\
\dot{R} & =\alpha_{3} R\left(1-\frac{R}{\left(k-k_{1}\right)}\right)\left(\frac{R}{k 2}-1\right)-\alpha_{4} R T
\end{aligned}\right.
$$

\section{Static solutions and their stability}

The proposed equations (4) are time-independent when $\dot{T}$ and $\dot{R}$ are simultaneously equal to zero.

$$
\left\{\begin{array}{l}
0=\alpha_{1} T\left(1-\frac{T}{k_{1}}\right)-\alpha_{2} R \\
0=\alpha_{3} R\left(1-\frac{R}{\left(k-k_{1}\right)}\right)\left(\frac{R}{k 2}-1\right)-\alpha_{4} R T .
\end{array}\right.
$$

Discussion about the stability of the solution is done according the values of the eigenvalues of Jacobian matrix,

$$
J=\left(\begin{array}{cc}
\alpha_{1}-2 T \frac{\alpha_{1}}{k_{1}} & -\alpha_{2} \\
-\alpha_{4} R & \alpha_{3}\left(-1+\frac{2 R}{k_{2}}+\frac{2 R}{k-k_{1}}-\frac{3 R^{2}}{k-k_{1}}\right)-\alpha_{4} T
\end{array}\right)
$$

- For $T=0, R=0$,

$$
J=\left(\begin{array}{cc}
\alpha_{1} & -\alpha_{2} \\
0 & \alpha_{3}
\end{array}\right)
$$

This solution (zero tourists and zero residents) is unstable because the eigenvalues $\alpha_{1}$ and $\alpha_{3}$ (the natural growth ratio of tourists and residents respectively) are real positive numbers. 
- For $T=k_{1}, R=0$,

$$
J=\left(\begin{array}{cc}
-\alpha_{1} & -\alpha_{2} \\
0 & -\alpha_{3}-\alpha_{4} k_{1}
\end{array}\right)
$$

In this case, stability depends on the sign of the parameters $-\alpha_{3}-\alpha_{4} k_{1}$. Describes a scenario without residents, being the city only for tourists, which we call "theme park".

- For $R \neq 0$ and $\dot{R}=0$ ), we obtain,

$$
T=\frac{\alpha 3}{\alpha_{4}}\left(\frac{k-k_{1}+k-2}{k_{2}\left(k-k_{1}\right)} R-1+\frac{R^{2}}{k_{2}\left(k-k_{1}\right)}\right)
$$

and a four degree polynomial, that,

- May not have any real solution. Then, there would be only two stationary solutions, the null and so-called "theme park".

- May have two real solutions and two complexes. There would be 4 stationary solutions.

- May have four real solutions. In total there would be 6 stationary solutions.

\section{Results and discussion}

There is some freedom in the choice of parameters and the results strongly depend on these values. The authors would like to emphasize the methodology and the discussion of the results, rather than the specific results, which have been understood as one of the possible scenarios. In our model, natural growth factors $\left(\alpha_{1}=0.08\right.$ and $\left.\alpha_{3}=0.025\right)$ are taken from the literature and previous works by the authors [11]. K-parameters are reasonable values for Barcelona, related to their capacity to hold residents and tourists $k=2 e 6, k_{1}=2 e 5, k_{2}=k_{1}$.

A bifurcation analysis have been done varying some meaningful parameters like $\alpha_{2}$ and $\alpha_{4}$ (starting values of $\alpha_{2}=0.00005, \alpha_{4}=0.0000002$ have been selected in order to study the range around the bifurcation points).

There are at least six real time independ solutions of the dynamical system that can be found solving the equations (4).

1. $T=0, R=0$. 
2. $T=k_{1}, R=0$.

3. $T=125.22, R=200225.43$.

4. $T=1130.11, R=1797963.20$.

5. $T=199210.99, R=1257434.16$.

6. $T=199533.68, R=744377.21$.

Figure 1 shows the time independent solutions for tourist and residents for a range of $\alpha_{4}$ values. The stationary solution $\mathrm{T}=200000, \mathrm{R}=0$ ("theme park") is also represented. Only the "theme park" solution stands to the right of the limit point $(\mathrm{LP}=2.2 \mathrm{e}-7)$. Between the branch point $(\mathrm{BP}=-1.2 \mathrm{e}-6)$ and limit point $(\mathrm{LP}=2.2 \mathrm{e}-7)$ there are two stable stationary solutions.

LP bifurcation is associated to the disappearance of sustainable equilibrium, leading to the extinction of a population. It usually appears when policy factors $\left(\alpha_{2}\right.$ and $\left.\alpha_{4}\right)$ grow too much in absolute value.

The existence of negative values of $\alpha_{4}$ can be understood as a positive effect of residents, increasing the affluence of tourists.
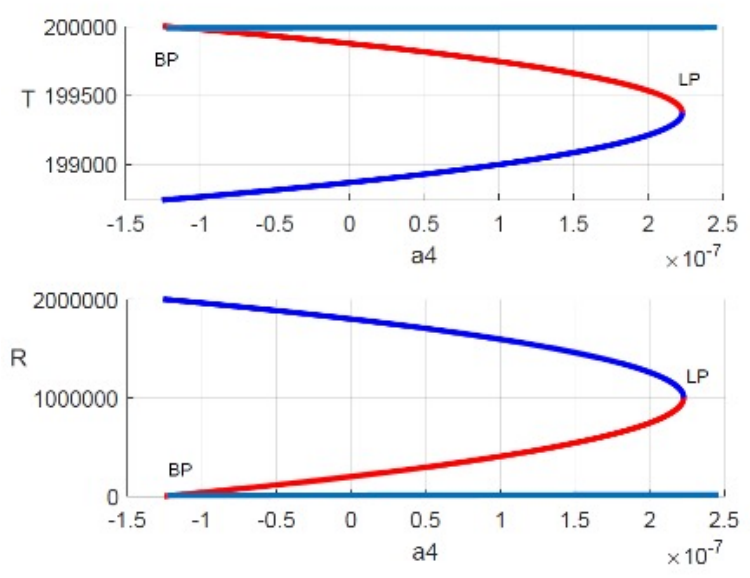

Figure 1. Time independent solutions for tourists $(\mathrm{T})$ and residents $(\mathrm{R})$ for a range of the parameter $\alpha_{4}$. BP stands for Branch Point and LP for Limit Point. Red and blue are for unstable and stable solutions respectively

Figure 2 shows the time independent solutions for tourist and residents for a range of $\alpha_{2}$ values. Limit points are $\mathrm{LP}=-0.0020048609$ and $\mathrm{LP}=0.0107725061$ 

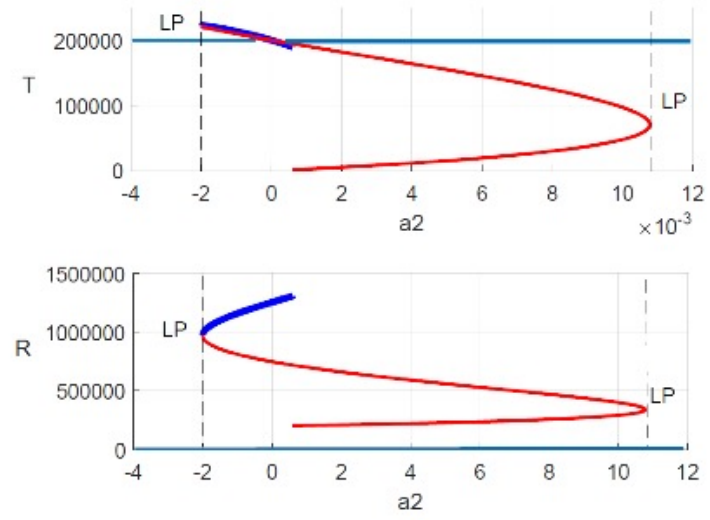

Figure 2. Time independent solutions for tourists $(\mathrm{T})$ and residents $(\mathrm{R})$ for a range of the parameter $\alpha_{2}$. BP stands for Branch Point and LP for Limit Point. Red and blue are for unstable and stable solutions respectively

\section{Conclusions}

The dynamic interaction between tourists and residents has been modeled through a nonlinear system of ordinary differential equations. The model takes into account the natural growth of both populations and external actions associated with political decisions and reaction actions of tourists and resident populations. Initially, the model has been applied to the city of Barcelona, but it will be necessary to do more future work to calibrate the parameters of this city.

The proposed equations describe the problem and the expected behavior, but the specific results must be taken with some caution because of the strong dependence of the associated parameters. It is a non-systematic study and the specific results must be understood as one of the possible scenarios. In this scenario, our results show that sustainability, that is, the positive values of the population of tourists and residents, is possible under the appropriate economic and social decisions.

Our bifurcation analysis varying the parameters associated to political and reacting actions have found several stable stationary solutions. The existence of Limit Points indicates that stationary solutions can not be found when policy factors grow beyond that point. There is also a stationary solution corresponding to zero residents. 


\section{Acknowledgments}

This work was partially supported by the Department of Applied Maths of the Universitat Politecnica de Catalunya

\section{References}

[1] Ajuntament Barcelona: Statistics and surveys. http: //ajuntament. barcelona.cat

[2] Gremi d'hotels de Barcelona. http://www . barcelonahotels.org

[3] Statista https://www.statista.com/topics/4156/tourism-in-barcelona/

[4] Servei de premsa http://ajuntament.barcelona.cat/premsa/tag/turisme/

[5] Lotka, A.J. Elements of mathematical biology. Dover Publications Inc. 1956.

[6] Volterra, V. Variazioni e fluttuazioni del numero dindividui in specie animali conviventi (in Italian), vol. 5, pp. 113. 1926.

[7] Brander, J.A., Taylor, M. S. The simple economics of Easter island: A Ricardo-Malthus model of renewable resource use. The American Economic Review, vol. 88, pp. 119-138. 1998.

[8] D'Alessandro, S. Non-linear dynamics of population and natural resources: The emergence of different patterns of development Ecological Economics 62 473481. 2007.

[9] Rinaldi S., Casagrandi R. A Theoretical Approach to Tourism Sustainability Ecology and Society. 2002

[10] Sinay L., Sinay L. A simple mathematical model for the effects of the growth of tourism on environment International Tourism Conference. Alanya, Turkey. 2006.

[11] Cholo I., Massana I., Olivar G., Prat, J.A., Trullols E. Bifurcations in a Model of Natural Resources and Human Activity. World Conference on Natural Resource Modeling, Barcelona. 2017.

Enric Trullols, Ph.D.: Universitat Politecnica de Catalunya/SARTI research group, department of Applied Maths, Jordi Girona 31, 08034 Barcelona, Spain (enric.trullols@upc.edu). The author presented this contribution at the conference in the special session "MegaCities: Nonlinear dynamics and networks" organized by G. Olivar.

Imma Massana, Ph.D.: Universitat Politecnica de Catalunya/SARTI research group, department of Applied Maths, Jordi Girona 31, 08034 Barcelona, Spain (immaculada.massana@upc.edu). 
Joana d'Arc Prat, Ph.D.: Universitat Politecnica de Catalunya/SARTI research group, department of Applied Maths, Jordi Girona 31, 08034 Barcelona, Spain (joana.darc.prat@upc.edu).

Josefina Antonijuan, Ph.D.: Universitat Politecnica de Catalunya/SARTI research group, department of Applied Maths, Jordi Girona 31, 08034 Barcelona, Spain (josefina.antonijuan@upc.edu).

Gerard Olivar, Ph.D.: Universidad Nacional de Colombia, Carrera 27 \# 64-60 Manizales, Caldas, Colombia (golivart@unal.edu.co). 